\title{
JACQUES ROGER HISTORIEN DES SCIENCES
}

Jacques Roger est mort le 26 mars 1990. Peu de mois s'étaient écoulés depuis la publication de sa grande biographie de Buffon, œuvre d'exigence et de «secrète sympathie", qui concrétisait des années de recherche patiente et qu'il aura vu saluée, à son grand bonheur, selon son mérite. Jacques Roger était né le 24 octobre 1920. Quoiqu'il occupât une position centrale dans l'histoire des sciences et qu'il illustrât dans la communauté internationale la meilleure tradition française, il n'était, de formation, ni historien, ni scientifique, ni philosophe mais agrégé de lettres classiques (1943). Jacques Roger a été professeur aux lycées de Douai, de Lille, de Suresne et au collège Stanislas entre 1943 et 1951, avant de devenir chargé de recherche au C.N.R.S. de 1951 à 1953 puis assistant de littérature française à la faculté des lettres de Poitiers. Si son premier ouvrage fut un Panorama du xvII siècle français, publié en 1962, il avait néanmoins déjà « découvert sa voie » lors d'entretiens qu'il eut, dès 1945, avec Antoine Adam. En " aboutissement lointain, mais direct » de cette seconde vocation, il soutint en 1963, sous la direction de René Pintard, sa thèse de doctorat: Les Sciences de la vie dans la pensée française du xиII' siècle, une somme d'érudition inégalée en histoire des idées. En 1964, il devint professeur, puis doyen de la faculté des lettres de Tours, avant d'être nommé professeur à la Sorbonne (1969) et directeur d'études à l'École des hautes études en sciences sociales (1982).

Successeur de Henri Berr à la direction du Centre international de synthèse (1978), directeur du Centre Alexandre-Koyré jusqu'en décembre 1989, Jacques Roger défendit toujours le concept d'une histoire intellectuelle générale contre les cloisonnements disciplinaires. Pour lui, l'unité du savoir dans les œuvres du passé impliquait qu'on oublie, pour sa restitution, les partages trop modernes entre idéologies et savoirs positifs. Pas plus qu'elle n'existait au ciel des idées, la science empirique ne lui semblait clôturée sur ses évidences axiomatiques : 
« on a trop souvent une certaine image de la science comme d'une activité absolument abstraite, qui ne subirait pas l'influence des courants intellectuels du monde dans lequel le savant vit.

Nous savons très bien que cette idée est fausse et que le savant, comme tous les intellectuels, vit dans un certain milieu historique très précis " !.

La division sciences/idéologies, "commode au premier abord", ne résistait pas à l'analyse attentive. Aussi bien tiendra-t-il deux propositions critiques qui ordonnaient toute sa recherche. D'une part,

"l'histoire des sciences est devenue une discipline à part entière, et l'historien des sciences a compris que son rôle, comme celui de tout historien, est de comprendre, non de juger, et que, selon la formule de Lucien Febvre, le seul péché capital en histoire, c'est l'anachronisme ${ }^{2}$.

D'autre part, il affirmait que les grandes fresques épistémologiques à caractère récurrent ou judicatoire relevaient d'une histoire jugée, formellement téléologique, ou selon son mot " arrogante ». Cette arrogance, il la dira " insupportable ". Elle impliquait un « ton de blâme moral " à l'égard des savants du passé dont les travaux avaient à leur tour permis qu'ils fussent dépassés ${ }^{3}$.

Militant pour une histoire historique des sciences ${ }^{4}$, Jacques Roger publiait souvent ses convictions en prenant d'égales distances vis-à-vis de la philosophie et de la reconstruction par les scientifiques des " progrès " de leur discipline. Car «l'historien n'est pas un philosophe $»^{5}$ et, sur ce chapitre des motivations, il reprochait aux scientifiques un « attachement sentimental " pour la recherche des " précurseurs" où il voyait une " pieuse mystification ». "Cette histoire étudie la science d'autrefois non pas pour ce qu'elle est, mais pour ce qu'elle doit devenir avec le temps,

1. Jacques RoGER, « La crise scientifique avant Galilée », in Cultura e Società nel Rinascimento. Tra Riforme e Manierismi, V. BrancA, C. Ossola, eds, Florence, Olschki, 1984, p. 1.

2. ID., Buffon. Un philosophe au Jardin du roi, Paris, Fayard, 1989, p. 11.

3. ID., « Darwin en France », Annals of Science, vol. 33, 1976, p. 484.

4. ID., "Per una storia storica delle scienze", Giornale critico della filosofia italiana, anno LXIII (LXV), fasc. III, 1984, p. 285-314. Cet article important situe la recherche de l'historien des sciences par rapport à l'épistémologie et la sociologie des sciences dans une perspective essentiellement française - au moins pour la philosophie, cf. ibid., p. 299-300 : « uno storico poco addentro alla situazione francese della storia delle scienze si meraviglierà forse di una cosi lunga discussione, o riassunto, per lui superfluo, di verità così evidenti. Ma la storia epistemologica delle scienze ha esercitato troppo a lungo in Francia il suo impero intellettuale ed istituzionale perché uno storico francese delle scienze non provi il bisogno di situarsi rispetto ad essa ». Cet examen critique pour signifier que « il suo progretto è un altro, ed una storia delle scienze indipendente non può sottomettersi alla prospettiva dell'epistemologia. "

5. J. Roger, in Structuralisme et marxisme, Paris, U.G.E., « 10/18 », 1970, p. 223. 
c'est-à-dire précisément pour ce qu'elle n'est pas encore ». Elle avait encore ce défaut de méthode d'infantiliser les auteurs passés, transformés en « fantaisistes », naïfs et « ridicules fantoches », porteurs d'une pensée " inachevée, illogique ». Par un retournement saisissant et novateur, Jacques Roger, avant Michel Foucault, réclamait pour eux le bénéfice d'une lecture anthropologique :

«Au moment où les ethnologues réhabilitent la "pensée sauvage", il doit être permis à l'historien de dénoncer lui aussi l'illusion du " primitivisme ", et de demander le respect pour cette autre "pensée sauvage" que l'on appelle communément " préscientifique " $" 6$.

Non sans à-propos dialectique, il remarquait alors que cette pensée pouvait souffrir rétrospectivement d'être « au contraire trop achevée et trop logique ${ }^{7}$, au même titre que certaines de ces structures formelles qui, de leur lointain passé, nous sont devenues « aussi étrangères que des langues mortes $"{ }^{8}$.

Il existait pourtant des images archétypales, à caractère transhistorique, qui semblaient renaître à intervalles irréguliers. Jacques Roger a souvent interrogé, dans ses écrits et ses enseignements, ce retour foncier des métaphores et des images valorisées dont il ne refusait ni le rôle actif dans la créativité scientifique, ni l'origine vraisemblablement psychologique. Mais il lui rebutait qu'une " psychanalyse » de la connaissance occultât l'histoire et le contexte de leur énonciation :

« il y a en nous des archétypes mais je ne sais pas si cela suffit, car dans certains cas nous nous heurtons à une réalité historique rigoureusement étrangère, par exemple la vision aristotélicienne du cosmos. De véritables mutations de l'imagination et de la manière dont nous ressentons le monde se produisent $"{ }^{9}$.

$\mathrm{Si}$ les productions de l'esprit humain se partageaient également selon des couples d'opposés, le continu et le discontinu, le mécanisme et le vitalisme, le solide et le fluide, le hasard et la finalité, etc., rien n'indiquait que ces schémas imaginaires fussent, dans l'absolu, immuables. D'abord, les mots et les images auxquels on les associe aujourd'hui ne devaient pas faire illusion sur la différence des réalités qu'ils recouvraient historique-

6. ID., "Réflexions sur l'histoire de la biologie (xvII $-\mathrm{xvIII}^{e}$ siècle): problèmes de méthodes ", conférence de février 1964, Revue d'histoire des sciences, t. XVII, janv.-mars 1964, p. 31. Les citations précédentes sont extraites du même texte, p. 27 et 29.

7. Ibid., p. 29.

8. J. ROGER, " Lecture des textes et histoire des idées ", in Les Chemins actuels de la critique, Georges Poulet, éd., colloque de 1966, Paris, U.G.E., «10/18 », 1968, p. 284.

9. Ibid., p. 291. 
ment. Jacques Roger en fit la démonstration à partir du concept de "germe " dont il critiqua l'interprétation bachelardienne ${ }^{10}$. Ensuite, l'histoire seule rendait raison de leur prédominance à telle ou telle date : " on fait parfois remonter l'idée d'une chaîne des êtres jusqu'à Aristote. C'est pourtant au début du $x^{\prime} I^{e}$ siècle qu'elle devient un élément important de la réflexion des philosophes et des naturalistes $" ~ "$. Enfin, du fait q̨u'il n'est pas unanime dans la communauté scientifique, l'accord scellé sur la priorité des images devait être à son tour questionné dans la chronologie de son instauration, de son invalidation ou de sa transformation. Cet accord manifestait un problème historiographique plutôt que sa solution. «L'un des problèmes de l'historien des idées est celui des thèmes fondamentaux. On a le sentiment quand on les voit paraître qu'ils sont en effet très anciens, mais on peut se demander pourquoi tel moment de l'histoire leur insuffle une force nouvelle ${ }^{12}$.

Jacques Roger croyait et démontrait que les auteurs du passé avaient, souvent de manière indirecte, produit des innovations d'ensemble concernant la vision de la nature qui préparaient leur réfutation posthume. Ils l'avaient néanmoins amorcée ou préfigurée : "il est assez fréquent dans l'histoire des sciences que les grandes révolutions théoriques commencent par une situation où celui qui présente une nouvelle théorie n'est pas capable de la démontrer $"{ }^{13}$. L'exemple de Buffon, topique et plus encore "admiré ${ }^{14}$, fut souvent requis pour cette démonstration. L'intendant du Jardin du roi n'était pas transformiste mais il avait, " plus que personne au xviII siècle ", contribué " à rendre possible une théorie de l'évolution en transformant l'esprit de l'histoire naturelle $" 15$. Sans avoir

« ni inventé, ni pressenti le second principe de la thermodynamique [... il avait] créé un modèle original où quelques concepts fondamentaux, lois, hasard, ordre, histoire, énergie, sont liés entre eux d'une manière très particulière. Buffon ne sait pas encore clairement nommer ces concepts, mais le rôle qu'ils jouent dans la science moderne nous aide à les reconnaître et à découvrir l'originalité du système de pensée de Buffon ${ }^{16}$.

10. Cf. « Réflexions sur l'histoire de la biologie ", art. cit. supra n. 6, p. 33 sq.

11. J. Roger, "L'histoire naturelle au xviI" siècle : de l'échelle des êtres à l'évolution ", Bulletin de la Société zoologique de France, vol. 115, 3, 1990, p. 245-254, en part. p. 246.

12. Cf. "Lecture des textes et histoire des idées", in op. cit. supra n. 8, p. 292.

13. J. Roger, "Le transformisme de Lamarck", in Le Darwinisme aujourd'hui, Paris, Seuil, 1979, p. 23.

14. ID., in Buffon, Les Époques de la Nature, J. Roger, éd., Paris, Ed, du Muséum, 1962, p. v: " nous sommes de ceux qui croient que la sympathie est encore la meilleure méthode historique." "

15. ID., "Buffon et le transformisme ", La Recherche, 138, 1982, citation p. 1254.

16. ID., " Énergie, ordre et histoire dans la pensée de Buffon », Histoire et nature, 19-20, 1981-1982, p. 54-55. 
Jacques Roger pouvait dès lors, et sans anachronisme, restituer au passé le poids de ses images mythiques, anticipées ou porteuses d'un sens à venir. Il en administra la preuve convaincante à propos de la notion de " matière vivante $"{ }^{17}$ ou à propos du vitalisme, doctrine consubstantielle aux Lumières qui contribua pourtant activement à en détruire l'esprit, « en aidant au succès de la philosophie romantique » ${ }^{18}$. L'examen des « conséquences » de semblables intuitions valait autant pour éclairer la démarche scientifique. En règle générale, les conditions intellectuelles de l'apparition puis de la diffusion d'une idée force, capable d'ordonner la recherche, se révélaient pour lui à la fois dynamiques, complexes et architectoniques. Dynamiques parce qu'elles relevaient d'un processus de longue durée basé sur la force de persuasion et d'affirmation, la capacité d'harmoniser des idées, des institutions qui les discutent et les portent plus avant; complexes car l'interaction des acteurs multiples, venus d'horizons disciplinaires et épistémiques variés ou contradictoires, ne permettait pas à l'historien de conclụre à la nécessité d'un devenir du savoir en dehors de sa confrontation avec l'expérience de la nature, les mentalités du groupe social, et avec les traditions scientifiques antérieures; architectoniques parce qu'on ne pouvait les dissocier de ce qui forme l'outillage mental et l' « univers intellectuel» d'un chercheur ou d'une communauté de travail en quête d'un statut académique : « È facile opporre la fisica aristotelica e quella di Newton, o la chimica flogistica e quella dell'800. Ma la “ rivoluzione chimica" legata al nome di Lavoisier è un fenomeno complesso, durato decenni e coinvolgendo tante personalità e opere scientifiche spesso contraddittorie $"{ }^{19}$.

De là bien des paradoxes, souvent soulevés par l'historiographie de Jacques Roger : ce ne sont pas les philosophes matérialistes qui ont formulé l'hypothèse du transformisme mais les créationistes d'orientation leibnizienne qui ont requis des Nouveaux essais sur l'entendement humain l'idée d'un " dynamisme orienté réalisateur d'une histoire " de la vie, partie de la volonté divine ${ }^{20}$. C'est d'une crise de la science classique et d'une incertitude fondamentale sur l'essence des choses qu'est née la science moderne ${ }^{21}$. C'est en faisant entrer «l'âme dans l'histoire natu-

17. ID., "Chimie et biologie : des "molécules organiques" de Buffon à la "physicochimie " de Lamarck ", History and Philosophy of the Life Sciences, vol. 1, 1, 1979.

18. ID., "Science et Lumières ", Revue de l'Université de Bruxelles, 2-3, 1972, p. 165.

19. Cf. « Per una storia storica delle scienze », art. cit. supra n. 4, p. 293-294.

20. J. Roger, "Les conditions intellectuelles de l'apparition du transformisme ", in Epistémologie et marxisme, Paris, U.G.E., «10/18 », 1972, p. 99-114.

21. Cf. « La crise scientifique avant Galilèe», in op. cit. supra n. 1, p. 18-19. 
relle » que Buffon, cartésien anachronique ou au moins tardif, a rendu possible une science de l'homme riche d'avenir ${ }^{22}$.

Parmi bien d'autres contresens accrus par le rationalisme normatif moderne, Jacques Roger aimait à rappeler que le "mythe du conflit nécessaire entre le christianisme et la science " était né au XIX ${ }^{\mathrm{e}}$ siècle seulement, alors que la science acquérait le « formidable pouvoir intellectuel et social qu'elle possède aujourd'hui " ${ }^{23}$. De même il serait " puéril " de s'imaginer que les naturalistes du xvi ${ }^{\mathrm{e}}$ siècle, attachés aux formes traditionnelles du savoir, aient « tourné délibérément le dos à la nature et aux faits, et considéré leur science comme une pure gymnastique logique $"{ }^{24}$. Il fallait dès lors redonner au passé toute la densité de ses formules, de ses exigences, de ses controverses ou de ses enjeux intrinsèques. Ce programme d'érudition extrêmement novateur ordonna toute l'écriture de la

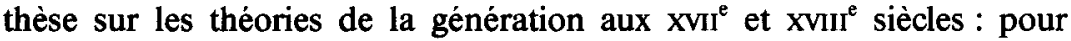
comprendre l'esprit des institutions ou la raison des survivances passéistes en balayant les caricatures inquisitoriales, Jacques Roger équilibrait les études à caractère morphologique et les approches prosopographiques. Alors qu'on prononçait l'effacement du sujet et la «mort de l'auteur » (M. Foucault), alors qu'on signifiait qu' « en histoire des sciences les noms propres ne devraient avoir que valeur d'index " ${ }^{25}$, Jacques Roger réaffirmait sereinement que la science est affaire d'individus engagés dans des conflits idéologiques datés et que la position sociale, la valeur humaine, " le tempérament intellectuel » des savants étaient aussi centraux pour l'évolution des doctrines que la construction pure des concepts. Non sans attendus polémiques, il publia son « plaidoyer pour l'auteur » au moment où la Nouvelle Critique des années 1960 s'efforçait d'oublier qu'un livre soit le fait d'un « homme vivant » : « allons plus loin, et demandons-nous s'il ne faudrait pas ressusciter un instant le

22. J. Roger, "Buffon et la théorie de l'anthropologie », in Enlightenment Studies in Honour of Lester G. Crocker, A. J. Bingham, V. W. Topazio, eds, Oxford, 1979, p. 253-262.

23. ID., " Le christianisme et la science moderne », in Le Grand atlas des religions, Paris, Encyclopaedia Universalis, 1988, p. 333.

24. ID., Les Sciences de la vie dans la pensée française du xvIIt siècle. La génération des animaux de Descartes à l'Encyclopédie, Paris, Armand Colin, $2^{\mathrm{e}}$ éd., 1971, p. 31.

25. Camille Limoges, La Sélection naturelle. Étude sur la première constitution d'un concept (1837-1859), Paris, P.U.F., 1970, p. 152. Cf. également, à titre indicatif, ces phrases prononcées par Michel Foucault lors du colloque « Georges Cuvier », Thalès, t. XIII, 1969, Revue d'histoire des sciences, t. XXXIII, 1,1970 , p. 88 : " je dois avouer que j'ai été mal à l'aise (et d'un malaise que je n'ai pas pu surmonter) lorsque, dans Les Mots et les choses j'ai mis en avant des noms. J'ai dit "Cuvier ", " Bopp", " Ricardo ", alors qu'en fait j'essayais par là d'utiliser le nom, non pas pour désigner la totalité d'une œuvre qui répondrait à une certaine délimitation, mais pour désigner une certaine transformation qui a lieu à une époque donnée et qu'on peut voir mise en cuvre, à tel moment et en particulier dans les textes en question [...] mon problème est de repérer la transformation. Autrement dit, l'auteur n'existe pas. » 
couple damné du psychologisme et de la biographie, frère et sœur abominables! $"{ }^{26}$. Dans l'unité d'une œuvre, la biographie de Buffon, publiée en 1989 , prolongeait ainsi les réflexions qui ouvraient la troisième partie des Sciences de la vie de 1963, où Jacques Roger montrait que la philosophie nouvelle des années 1745-1770 demeurait subordonnée à des « recherches individuelles qu'on ne pourrait étudier ensemble sans trahir leur originalité ${ }^{27}$.

En vérité, l'œuvre historiographique de Jacques Roger ne fait pas seulement retour sur les conditions individuelles, sociales et intellectuelles de l'élaboration des savoirs. Elle mesure exactement le poids des inerties et des stéréotypes dans l'expression de leur progrès. On trouve dans bien des analyses des mises à l'épreuve d'une conclusion portée naguère sur le statut des sciences de la terre au $x v^{e}$ siècle : " chaque tradition apporte ses habitudes mentales, et l'unanimité se fait plus facilement dans les croyances irraisonnées que dans les connaissances positives ${ }^{28}$. Toutefois, on se tromperait en qualifiant cette attitude de scepticisme alors qu'il s'agit d'une posture d'analyse. Aussi bien Jacques Roger peut-il affirmer, à propos de la sociobiologie, qu'elle rate doublement son objet, à la fois comme avatar d'un scientisme " périmé " et comme synthèse " prématurée » des acquis majeurs de la théorie de l'évolution. Il n'en souligne pas moins qu'elle

« marque le lieu où peut et doit se faire une science générale du vivant. [...] En nous rappelant un peu brutalement notre origine animale, la sociobiologie réagit utilement contre l'irréalisme des spécialistes d'anthropologie culturelle, qui ont voulu tout ramener à la culture et à la société, et contre l'angélisme des moralistes et des utopistes. L'homme n'est pas une tabula rasa, une argile malléable dont on peut faire n'importe quoi ${ }^{29}$.

De telles tensions révéleront, dans l'après-coup de leur contestation, leur capacité critique. Ce qui est un temps perçu en termes négatifs peut gagner en valeur positive : " on a beaucoup critiqué Buffon pour avoir introduit des éléments "psychologiques" dans sa description des ani-

26. Cf. " Lecture des textes et histoire des idées ", in op. cit. supra n. 8, p. 281. L'intérêt historiographique de la biographie des savants est justifié par J. Roger dans un autre texte, " The DSB [ie. : The Dictionary of Scientific Biography, Ch. C. Gillispie, ed.] : A review symposium ", ISIS, vol. 71, 1980, p. 649-650; cf. également son compte rendu du livre de Dorinda Outram, Georges Cuvier, in History and Philosophy of the Life Sciences, vol. 9, 1987 , p. $369-371$.

27. Cf. Les Sciences de la vie, op. cit. supra n. 24, p. 458.

28. J. Roger, "Les sciences de la terre ", in Histoire générale des sciences, René TATON, éd., Paris, P.U.F., 1969, t. II, p. 120. Cf. Les Sciences de la vie, op. cit. supra n. 24, p. 8.

29. ID., « La sociobiologie est-elle à l'heure? », Revue de synthese, III ${ }^{e}$ S., t. CI, 97-98, 1980, p. 154. 
maux. Nous pouvons considérer aujourd'hui que cette introduction de l'éthologie constituait un élargissement important de l'histoire naturelle ${ }^{30}$.

C'est là le prix à payer pour penser ensemble la persévérance de l'esprit humain dans sa quête de sens, ce prodigieux effort collectif pour ordonner le chaos des phénomènes dont la recherche fit le fond de l'humanisme de Jacques Roger, et la complexité déroutante de son accomplissement. Cette complexité est de structure, non d'accident. Elle ressortit à des " mentalités " ${ }^{31}$ qui n'ont rien d'uniforme, à l'infrastructure sociale, philosophique, politique, voire religieuse de la science, à l'ordre matériel même qui régit ses lieux professionnels, à la construction de ses concepts et des lois qui les articulent. Pour qu'une science géologique s'émancipe d'une "physique " universelle, il a fallu que la science se dote de laboratoires : « ce n'était pas rien : c'était peut-être tout ». De même « il n'est pas exagéré de dire que le microscope a changé pour les savants la face du monde $"{ }^{32}$. Aux causes minimes en apparence, les grands effets. Le conflit des modèles scientifiques dans l'unité synchronique d'une époque donnée se redouble de problèmes de méthodes et d'expérimentations qui trouvent des issues partielles et discutées dans l'enceinte des académies savantes. Pour Jacques Roger, historien des modèles en relation antagonique plus que des structures, la continuité du savoir exige des discussions, des accommodements subtils et pour tout dire des infidélités aux pères fondateurs qui font l'histoire même ${ }^{33}$. Pour une période, pour un auteur choisis, de telles transactions définissaient un champ de possibilités souvent concurrentes. A travers ses «images collectives ", l'ensemble finit cependant par dessiner un " paysage scientifique " capable de contraster l'ombre et les lumières. Sur ce fond de relations conflictuelles, Jacques Roger ne balançait pas à penser que chaque époque témoignait selon ses mots d'une " cohérence propre " ${ }^{34}$, d'un « écosystème intellectuel » ${ }^{35}$, de « structures profondes de la sensi-

30. ID., " Histoire naturelle et biologie chez Buffon », in Lazzaro Spallanzani e la biologia del Settecento, Giuseppe Montalenti, Paolo Rossi, eds, Florence, Olschki, 1982, p. 357.

31. J. Roger entendait sous ce mot les propositions jugées par soi évidentes, les conventions non écrites, les réflexes conditionnés constituant « le non-dit du discours scientifique admis dans une communauté particulière ", cf. J. RoGER, « Histoire des mentalités : les questions d'un historien des sciences ", Revue de synthese, III ' S., t. CIV, 111-112, 1983, p. 269 275.

32. Cf. Les Sciences de la vie, op. cit. supra n. 24, p. 183.

33. Voir les remarques sur les relations qu'entretenait Lamarck avec l'idée de la chaîne des êtres, in art. cit. supra n. 11 , p. 254.

34. Cf. «Objectivité et historicité de la pensée scientifique », in op. cit. supra n. 5, p. 222 $s q$.

35. J. ROGER, « Histoire de la science ou des sciences? Une histoire ou des histoires des sciences ", Séminaire d'épistémologie et d'histoire des sciences, Paris, Institut national agronomique, Paris-Grignon, 1986-1987, p. 37. 
bilité et de l'imagination " ${ }^{36}$, dotées d'une logique et d'un univers de sens dont les résistances et l'altérité forçaient la curiosité. Cette altérité n'était pas un vain mot non plus que cette curiosité d'homme. Rappelant que Walter Pagel n'avait pas cherché à faire la synthèse de la pensée paracelsienne, J. Roger soulignait les difficultés attachées au dialogue de deux cultures linguistiquement et donc mentalement incommensuiables :

" C'est peut-être que cette synthèse est impossible dans la langue de notre temps. Les réalités que Paracelse désigne sont d'un autre ordre que celles que nos concepts recouvrent, et les liens qu'il met au jour relèvent d'une autre logique. Pour comprendre Paracelse, pour embrasser son œuvre, il faut sans doute renoncer à le traduire et tenter de suivre sa pensée au niveau qui est le sien, celui des images et des symboles $"{ }^{37}$.

S'il recherchait la plénitude du sens qui définit l'esprit d'une époque sans occulter les contradictions de ses « familles intellectuelles", Jacques Roger affirmait également le poids des héritages conceptuels dans l'évolution et la diffusion des savoirs. Éclairer le texte savant supposait sans doute l'herméneutique des œuvres, mais la réflexivité s'y trouvait également engagée. L'approche structurale n'y suffisait pas. Trois arguments de type épistémologique justifiaient l'attention qu'il portait aux pratiques de la lecture comme à son histoire.

1. L'historien est dans l'histoire, au même titre que l'auteur étudié. "L'histoire est au cœur même de l'œuvre, non seulement si nous y cherchons un homme, mais encore si nous la considérons en elle-même et sans auteur. Est-il besoin de montrer que l'histoire est aussi dans le lecteur? $"{ }^{38}$.

2. Ceci déduit de cela, le texte n'est pas indépendant de ses lecteurs. Il change avec eux.

3. Il y a une histoire évolutive des modes d'administration de la preuve, de l'usage réglé des métaphores, de la construction de l'objet. D'où ces deux conséquences : d'un côté, « le jeu réciproque de la forme et de la matière nous apparaîtront beaucoup plus clairement si nous examinons leurs variations au long de l'histoire ". D'un autre côté, ces interactions réciproques, ces effets pragmatiques et stratégiques dialectisaient (en les ruinant partiellement) les thèses naguère opposées de l'histoire « internaliste" et de l'histoire "externaliste " des savoirs scientifiques ${ }^{39}$. Non

36. Cf. « Lecture des textes et histoire des idées ", in op. cit. supra n. 8, p. 287.

37. J. RoGER, "Quelques années d'études paracelsiennes en France", Archives internationales d'histoire des sciences, 92-93, 1970, p. 227.

38. Cf. " Lecture des textes et histoire des idées ", in op. cit. supra n. 8, p. 285.

39. Ibid., p. 285 ; cf. également « Per una storia storica delle scienze ", art. cit. supra n. 4, p. 311-313. 
sans humilité professionnelle, Jacques Roger soulignait ainsi la limite de l'objectivité historienne comme son idéal régulateur.

L'étude lexicographique du vocabulaire scientifique lui apparaîtra une approche privilégiée de ces déterminismes de plus longue durée. Intéressé à l'explicitation des contenus notionnels changeants du lexique et convaincu que "l'idée même que la science doit être une langue bien faite n'est apparue ni spontanément ni immédiatement $"{ }^{40}$, il animera ainsi, à partir de 1979, un groupe de recherche transdisciplinaire dont l'une des priorités éditoriales sera la réalisation d'un Dictionnaire historique $d u$ vocabulaire scientifique, actuellement en chantier.

Jacques Roger n'était pas un théoricien des grandes discontinuités ou des " coupures » épistémologiques, mais un analyste des déplacements subtils, inscrits dans la durée. Il admettait avec réticence des généralités hâtives sur l'épistémè de l'âge classique, la «pensée » des Lumières ou la «vision » romantique de la nature. « De même qu'un tournant n'interrompt pas une route, mais pourtant l'oriente dans une autre direction, de même un tournant historique ne rompt pas la continuité de l'histoire... $"{ }^{41}$. Contrairement à Michel Foucault qui avait popularisé par sa philosophie épistémique la notion d'une « naissance » abrupte de la biologie au début du $x x^{e}$ siècle, il s'attachait à un mouvement scientifique séculaire, sans origine absolue :

« La naissance de la biologie, en tant que science particulière des êtres vivants, n'a pas été un phénomène instantané, qui aurait brutalement bouleversé la répartition des connaissances à la fin du xvir' siècle. C'est le résultat d'un processus complexe, qui commence à se manifester dès le début du siècle, et d'abord comme réaction à la situation faite aux êtres vivants par le mécanisme du xviI ${ }^{e}$ siècle ${ }^{42}$.

Aussi bien, Jacques Roger admettait-il que le passage d'une époque à une autre procède par deux types de transitions, soit qu'un système se dégrade insensiblement de l'intérieur par tout un jeu de tensions et de contradictions, soit que les éléments d'un système nouveau s'expriment dans les termes du système ancien : « il y a des structures, mais elles ne sont jamais en parfaite cohérence, en parfait équilibre. C'est par ce

40. J. Roger, “Avant-propos », in Transfert de vocabulaire dans les sciences, volume préparé par Martine Groult, sous la dir. de Pierre Louis et Jacques Roger, Paris, Ed. du C.N.R.S., 1988 , p. 7.

41. Cf. " Lecture des textes et histoire des idées ", in op. cit. supra n. 8, p. 298.

42. Cf. « Chimie et biologie », art. cit. supra n. 17, p. 43 et J. RoGER, « L'Europe savante. 1700-1850", in Les Savants genevois dans l'Europe intellectuelle du XVIf au milieu du XIX' siècle, Jacques Trembley, éd., Genève, Ed. du Journal de Genève, 1987, p. 47 : « dans les sciences de la vie et de la terre, on observe à la fois la même effervescence et la même continuité entre la fin du xviI ${ }^{e}$ siècle et la première moitié du XIX ${ }^{e}$." 
manque de stabilité que la structure glisse, se déforme et qu'une autre structure apparaît $"{ }^{43}$.

Sans d'ailleurs se refuser à « interpréter à sa manière cette floraison de " discours de la méthode" " ${ }^{44}$, Jacques Roger gardera vis-à-vis du structuralisme des années 1960 un certain recul... proprement historiographique. Le structuralisme lui paraissait participer d'une de ces scansions historiques où le discontinuisme l'emportait sur le continuisme. Interrogeant cet effet de contexte, il lui assignera ses raisons nécessaires :

« la rationalité de notre siècle a appris à prendre en compte ces moments où soudain tout change et où rien n'est plus prévisible, dans l'histoire ou dans la physique. L'histoire discontinuiste de Michel Foucault rejoint ici la théorie des catastrophes de René Thom et la théorie de l'ordre par fluctuations d'Ilya Prigogine " ${ }^{45}$.

Selon Michel de Certeau, son grand ouvrage sur les sciences de la vie au XviI ${ }^{\mathrm{e}}$ siècle fournit aux Mots et les choses de Michel Foucault la matière de son érudition ${ }^{46}$. Pourtant Jacques Roger soupçonnait qu'à valoriser ainsi l'ordre des structures, on cherchât à évacuer la dimension temporelle du savoir: "que l'on ait récemment ressuscité Cuvier témoigne sur nous, non sur lui. Notre science n'est plus newtonienne et nos idéologies voudraient bien ne plus être historiques $"{ }^{47}$. A propos de Linné, réhabilité tardivement, il précisait cette «tentation métaphysique » : « l'historiographie récente l'a fait sortir, comme Cuvier, du purgatoire de l'histoire : par un effet de "récurrence", le rationalisme moderne a rendu historiens et philosophes plus attentifs à une pensée qui structure le réel en soulignant les discontinuités $"{ }^{48}$.

Le premier reproche méthodologique adressé à de telles récurrences, c'est qu'elles mutilent les auteurs étudiés, « qu'elles découpent arbitraire-

43. Cf. " Lecture des textes et histoire des idées ", in op. cit. supra n. 8, p. 296 ; « Per una storia storica delle scienze ", art. cit. supra n. 4, p. 313-314.

44. Cf. "Réflexions sur l'histoire de la biologie", art. cit. supra n. 6, p. 40. En fait, Jacques Roger affectionnait et cultivait, par respect de la recherche, une « épistémologie concordataire ", cf. "Lecture des textes et histoire des idées ", in op. cit. supra n. 8, p. 293 : " toute méthode qui permet d'étudier un objet passé, même si cette méthode se veut intemporelle, est une aide apportée à l'histoire. Telle méthode structuraliste, qui refuse la diachronie pour fixer un moment, l'explorer entièrement dans sa synchronie, est extrêmement précieuse aux historiens. " J'emprunte le concept d'épistémologie « concordataire » à Georges Canguilhem, non sans lui donner un contenu différent, of. « Sur une épistémologie concordataire ", in Hommage à Gaston Bachelard, Paris, P.U.F., 1957, p. 3-12.

45. J. Zoger, "Actualité de la science du xvil siècle ", in Destins et enjeux du xhI siècle, Paris, P.U.F., 1985, p. 269.

46. Michel DE CERTEAU, Histoire et psychanalyse entre science et fiction, Paris, Gallimard, 1987 , p. 15.

47. J. Roger, «Connaître la terre », Critique, 327-328, 1974, p. 803.

48. ID., « Linné et l'ordre de la nature ", La Recherche, 86, 1978, p. 182, 185. 
ment dans une cuvre du passé ce qui convient et ce qui ne convient pas à nos connaissances actuelles et, ce faisant, elles détruisent la logique interne des systèmes que l'on étudie $"{ }^{49}$. Le second reproche retourné contre l'épistémologie des discontinuités était sans doute plus réaliste, voire matérialiste. A l'instar de la philosophie des Lumières, le rationalisme contemporain risquait toujours d'idéaliser l'aventure du Concept et les moments de grande émergence de la science ${ }^{50}$, alors que la recherche de la vérité, par nécessité contingente, apparaissait à l'historien comme "l'humble résultat du long travail des hommes »" ${ }^{51}$. Par ailleurs, l'histoire prouvait suffisamment que la puissance d'affirmation d'une théorie nouvelle ne tient pas toute dans sa forme conceptuelle ${ }^{52}$. Sur l'exemple de Haeckel, J. Roger montra qu'une doctrine novatrice, comme l'était, en 1859 , l'évolutionnisme darwinien, fut soutenue «plus par des raisons idéologiques que par des raisons scientifiques $"$ " 33 . Il s'avérait que la raison gagne parfois grâce à de mauvaises raisons et que, tous blocages considérés, « ce n'est pas la raison scientifique qui règle l'avenir d'une théorie, au moins dans le court et le moyen terme ${ }^{54}$.

Ayant pris le parti d'une histoire intellectuelle des sciences, attachée à l'explicitation des déterminations de type culturel qui interagissent dans l'exercice d'une science normale comme dans sa réception, Jacques Roger supposait, en une formule qui résumait la perception qu'il avait de l'objet historiographique, des « structures mobiles " ${ }^{55}$. Paradoxe réel qui désignait sans doute la spécificité épistémologique du métier d'historien des sciences et sa difficulté : comment penser l'acquis de la science, son contenu de vérité parfois définitif, dans ses conditions relatives de formation et de transformation :

« la circulation du sang a été découverte par un homme très largement aristotélicien. Elle a donné lieu au Xvir siècle à un développement très important

49. Cf. « Histoire naturelle et biologie chez Buffon », in op. cit. supra n. 30, p. 359.

50. Cf. "Lecture des textes et histoire des idées ", in op. cit. supra n. 8, p. 291 : « Foucault dans Les Mots et les choses considère ces mutations comme un donné que ses méthodes d'archéologie ne permettent pas encore de réduire."

51. J. Roger, « La lumière et les Lumières ", Cahiers de l'Association internationale des Etudes françaises, 20, mai 1968, p. 177.

52. Cf. "Réflexions sur l'histoire de la biologie ", art. cit. supra n. 6, p. 30 ; " La genèse même de ces découvertes durables n'est pas toujours aussi pure que pourrait souhaiter le savant moderne."

53. J. RoGer, « Darwin et le darwinisme ", Centre interdisciplinaire d'Étude de l'évolution des idées, des sciences et techniques, Orsay, dactyl., 1983, p. 74.

54. Cf. "Histoire de la science ou des sciences?", in op. cit. supra n. 35, p. 38 . Cf. J. Roger, "Darwin, Haeckel et les Français", in De Darwin au darwinisme : Science et idéologie, Yvette Conry, éd., Paris, Vrin, 1983, p. 149-165; ID., " Présentation ", Revue de synthèse, III' S., t. C, 95-96, 1979, p. 279-282.

55. Cf. J. Roger, in op. cit. supra n. 5, p. 227 et « Per una storia storica delle scienze ", art. cit. supra n. 4, p. 313. 
d'une pensée biologique mécaniste, mais il est évident que la circulation du sang fait partie, sans discussion possible, de la science contemporaine. [...] Il y a donc ce type d'acquisition définitive $"{ }^{56}$.

Néanmoins, et quoique « daté », l'acquis considéré relevait d'une histoire presque linéaire, sans point d'arrêt définitif. « La science n'est pas une production comme les autres, où les produits se périment au fur et à mesure. Les travaux de Pasteur, fa reste ", remarquait Jacques Roger dans un "plaidoyer pour la culture scientifique ", publié dans Le Monde en novembre 1989. Mise en perspective, la « découverte » devenait processus, programme de recherche : « the " discovery" of the ovule or of the spermatozoon is in fact an ininterrupted process which may have begun with Regnier de Graaf and Van Leeuwenhoek, but has not stopped since and has doubtless not ended ${ }^{57}$.

Ce problème d'ordre philosophique en dernière instance fut réglé par Jacques Roger au bénéfice d'une dialectique historique de la connaissance, dans les formes d'un humanisme de la conscience dont l'élucidation, au cas par cas, fut au principe de son enseignement et l'objectif fédérateur de son séminaire d'histoire de la biologie à l'Université de Paris I et à l'E.H.E.S.S. Il publia cette profession de foi en 1964 :

« Dans la mesure où la recherche scientifique est une tension permanente et donc intemporelle de la conscience de l'homme vers le monde qui l'entoure, elle peut être considérée, au plus profond de sa réalité psychologique, comme une donnée essentielle de l'homme, échappant à l'évolution historique, inaccessible à toute histoire. Mais cette démarche scientifique, cette tension de la conscience, se manifeste par un regard et s'exprime par un discours. Depuis le début de l'aventure humaine, ce regard s'est structuré, ce discours s'est chargé de toutes les émotions et de toutes les victoires de l'homme. Même si elle ne peut être qu'une analyse et une tentative d'explication de ces structures, de ces émotions et de ces découvertes, l'histoire de la pensée scientifique vaut encore largement la peine d'être faite ${ }^{58}$.

Le séminaire devait porter la marque constante de cet intérêt pour les manifestations changeantes de ce rapport de l'homme à la nature, à sa nature.

56. Cf. J. RoGER, in op. cit. supra n. 5, p. 225.

57. ID., "Two Scientific Discoveries : Their Genesis and Destiny ", in On Scientific Discovery, Mirko D. Grmek, Robert S. Cohen, Guido Cimino, éds, Dordrecht/Boston/Londres, D. Reidel Publishing Company, 1980, p. 233. Ce destin véritablement transhistorique et international de l'activité scientifique éloignera Jacques Roger du réductionnisme sociologiste illustré par le "programme fort " de David Bloor. Cf. " Per una storia storica delle scienze ", art. cit. supra n. 4, p. 300 sq. Les arguments sont résumés p. 307.

58. Cf. « Réflexions sur l'histoire de la biologie ", art. cit. supra n. 6, p. 40. 
Professeur enthousiaste et complice, Jacques Roger entretint un dialogue pluridisciplinaire avec des élèves venus de tous les horizons intellectuels. Les historiens et les philosophes, les littéraires et les scientifiques trouvaient en lui un interlocuteur passionné mais également un excellent connaisseur des développements les plus récents de la recherche dans leurs divers domaines de compétence. A partir de 1970, son séminaire représentera pour tous un grand lieu de convivialité et d'échanges.

Ses derniers travaux publiés qui portaient en particulier sur les présupposés de la sociobiologie, l'eugénisme et l'anthropologie de Buffon témoignaient directement d'une réflexion sur l'éthique de la science de l'homme et la responsabilité sociale du savant. En juin 1989, lors d'un colloque sur l'histoire de l'anthropologie, il rappela les thèses de Thomas Jefferson réhabilitant, contre Buffon et Cornélius de Pauw, la condition des Indiens, "pour l'honneur de la nature humaine " ${ }^{59}$. Jacques Roger revendiquait cet «honneur " qui lui faisait une règle d'existence. Cet humanisme militant et cette générosité nous le rendent plus absent encore.

Claude BLANCKaERT, Centre Alexandre-Koyré, C.N.R.S., Paris.

59. J. Roger, "Buffon, Jefferson et l'homme américain ", in Histoire de l'anthropologie : hommes, idées, moments, Claude BlanckaERT, Albert Ducros, Jean-Jacques Hublin, éds, $\mathrm{n}^{\circ}$ spécial des Bulletins et mémoires de la Société d'anthropologie de Paris, $\mathrm{n}^{\text {ele }}$ série, t. I, 3-4, 1989, p. 57-66. 